\title{
Breast-fed infants and their later cardiovascular health: a prospective study from birth to age 32 years
}

\author{
Satu Pirilä ${ }^{1 *}$, Mervi Taskinen ${ }^{1}$, Heli Viljakainen ${ }^{1}$, Outi Mäkitie ${ }^{1,2}$, Merja Kajosaari ${ }^{1}$, \\ Ulla M. Saarinen-Pihkala ${ }^{1}$ and Maila Turanlahti ${ }^{1}$ \\ ${ }^{1}$ Children's Hospital, University of Helsinki, Helsinki University Central Hospital, PO Box 705, 00029 HUS, Helsinki, Finland \\ ${ }^{2}$ Folkhälsan Research Center, Helsinki, Finland
}

(Submitted 24 April 2013 - Final revision received 22 August 2013 - Accepted 3 September 2013 - First published online 8 November 2013)

\section{Abstract}

The aim of the present study was to evaluate the impact of infant breast-feeding on cardiovascular risk in young adults. This unique study group involved 158 subjects (eighty-two females) originally collected prospectively at birth in 1975 and followed up to the age of 32 years. Frequent visits during the first year guaranteed the knowledge of the precise duration of breast-feeding. All infants received at least some breast milk. Participants were assessed for both individual cardiovascular risk factors (blood pressure, plasma lipids, homeostatic model assessment of insulin resistance and waist circumference) and the general clinical risk of cardiovascular events by calculating the Framingham risk score (FRS) and the metabolic syndrome criteria score (NCEP-ATPIII; National Cholesterol Education Program's Adult Treatment Panel III). Data on lifestyle factors were carefully collected. Linear regression analyses revealed that the effect of the duration of breast-feeding was not relevant ( 0.02 decrease in the FRS per one additional breast-feeding month; $95 \% \mathrm{CI}$ $-0 \cdot 19,0 \cdot 09$ ). Similarly, the effect of breast-feeding was minor on all of the individual cardiovascular risk factors. We used sex, physical activity, dietary fat and vitamin $\mathrm{C}$, smoking and alcohol consumption as covariates. Again, logistic regression analyses detected no significant impact of the duration of breast-feeding on the risk of the metabolic syndrome according to the NCEP-ATPIII (OR 0.95, 95\% CI $0 \cdot 8,1 \cdot 1)$. The strongest independent predictor for later CVD risk was male sex. In conclusion, in this prospectively followed cohort of young adults born at term and at weight appropriate for gestational age, the duration of breast-feeding did not have an impact on the accumulation of cardiovascular risk factors.

Key words: Breast-feeding: CVD: Metabolic syndrome: Blood pressure

CVD is the most prominent lifestyle-dependent cause of morbidity and mortality in the Western world (WHO Health Report 2002). Development of vascular changes is thought to originate already in the prenatal and postnatal periods ${ }^{(1-4)}$. Impaired fetal growth from the middle to the late phases of gestation leads to disproportionate or reduced size at birth, which is associated with an increased risk of cardiovascular events later in life ${ }^{(1,3)}$. Postnatal factors including infant feeding ${ }^{(5)}$, infant growth $^{(4)}$ and childhood nutrition $^{(6)}$ may also play major roles in programming cardiovascular changes.

Breast-feeding in infancy is a potential determinant of cardiovascular risk factors and later CVD ${ }^{(5,7-12)}$. Studies vary in the duration of follow-up and have differences in the definition of breast-feeding. The reported effect of breast-feeding on cardiovascular health later in life has been controversial, as some groups have reported favourable effects ${ }^{(5,12,13)}$, some have found no association ${ }^{(14-16)}$ and some have reported an inverse relationship ${ }^{(17)}$.

We previously evaluated the effect of breast-feeding on body composition $^{(18)}$. Our previous results suggested that breastfeeding has an indirect influence on body composition in adulthood. Longer duration of breast-feeding resulted in lower-fat accumulation during the first year ${ }^{(19)}$, and this was positively tracked to central adiposity at 32 years of age. In the present study, our primary aim was to evaluate whether longer duration of breast-feeding reduces the risk of cardiovascular events in adulthood. The present cohort consisted of 32-year-old adults with no diagnosed cardiovascular events. We analysed the impact of breast-feeding on blood pressure, lipid profile, vascular properties and calculated clustered cardiovascular risk scores: the Framingham risk score (FRS) ${ }^{(20,21)}$ and the metabolic syndrome criteria score (National Cholesterol Education Program's Adult Treatment Panel III, NCEP-ATPIII) ${ }^{(22)}$.

Abbreviations: CCA, common carotid artery; FRS, Framingham risk score; HOMA-IR, homeostatic model assessment of insulin resistance; IMT, intimamedia thickness; NCEP-ATPIII, National Cholesterol Education Program's Adult Treatment Panel III; PROBIT, Promotion of Breastfeeding Intervention Trial. 


\section{Subjects and methods}

The present study was conducted according to the guidelines laid down in the Declaration of Helsinki, and all procedures involving human subjects were approved by the Ethics Committee of the Helsinki and Uusimaa Hospital District in Helsinki, Finland. Written informed consent was obtained from all the study participants.

\section{Participants}

In 1975, 238 healthy, full-term Finnish newborn babies at the Helsinki University Central Hospital were recruited to participate in a study examining the role of milk feeding in the Fe status of infants ${ }^{(23-26)}$. According to the inclusion criteria, the participants' birth weight exceeded $3000 \mathrm{~g}$. The mothers were recommended to breast-feed for as long as possible and all babies received breast milk for at least 2 weeks. When breast milk became insufficient, mothers were instructed to use a home-prepared cows' milk formula or a commercial milk formula (Bona ${ }^{\circledR}$; Chymos Oy). All babies were introduced to solid food at 3.5 months with the same strict guidelines. The same physician (U. M. S.-P.) examined all the babies seven times during the first year: at 2 weeks and then at 1, 2, 4, 6, 9 and 12 months of age. Data on milk feeding and anthropometric measurements were collected at each visit. The mean duration of breast-feeding was $5 \cdot 2$ (SD 3.2) months, while, on average, in Finland in 1974, only $30 \%$ were breast-fed for 3 months or longer ${ }^{(27)}$. The mean age at the introduction of weaning milk was 3.6 (sD 3.1) months.

Between 2006 and 2009, all the participants from the original cohort were traced through the Population Registry Centre in Finland, and invited to a 32-year follow-up assessment. From the original cohort, 188 participants were reached and 158 consented to participate and were examined at the outpatient clinic at the Children's Hospital, Helsinki University Central Hospital, Finland ${ }^{(18,28)}$. The participation rate was $66 \%$ : women were more likely to participate (72\%) than men $(60 \%)$. The reasons for non-participation were lack of time or interest.

All participants underwent physical examination by one of the authors (S. P.). Data were collected with a structured questionnaire on various lifestyle factors, such as current and previous physical activity, alcohol consumption, smoking, medical history (therapy for dyslipidaemia or hypertension) and education. The survey was completed by an interview during the clinical visit. Dietary intake of nutrients was assessed by $3 \mathrm{~d}$ food records. A licensed dietitian analysed the records with a computer program (software AIVO 2000Diet32, version 1.4.6.2; AIVO Finland Ltd).

\section{Blood pressure}

Hypertension was defined as systolic blood pressure $>130 \mathrm{mmHg}$, diastolic blood pressure $>85 \mathrm{mmHg}$ or by the treatment for hypertension. Blood pressure was recorded twice within 5 min after a supine rest of at least 10 min with an automatic device (Dinamap, Critikon; GE Medical Systems) and with a cuff size appropriate for the participants' left upper arm. The average of two measurements was used.

\section{Ultrasonography}

Vascular properties of the carotid artery were assessed with ultrasound by using a B-mode ultrasound imager (Vivid 7; GE Vingmed AS) with 12L vascular probe (GE Medical Systems). All measurements were performed by one of the authors (S. P.) who was blinded to the participants' breastfeeding history. Due to technical problems, images of only sixty-nine patients were available for detailed analysis at the end of the study. These measurements were regarded representative for the whole cohort as no differences in characteristics between those with and without ultrasonic examination were found (see the Supplementary table, available online). The examination setting was standardised, and started at 08.00 hours in the test office. The participants were examined after an overnight fast, and a $4 \mathrm{~h}$ tobacco and coffee restriction. Measurement for the right common carotid artery (CCA) was obtained $1-2 \mathrm{~cm}$ above bifurcation. A simultaneous echocardiogram recording was stored. The image frames of three subsequent beats were saved at the end diastole (ECG Q wave), and then CCA far-wall intimamedia thickness (CCA-IMT) was measured and the mean value of the three measurements was used. Analyses were performed from digitally stored data with semi-automated analysing software AMS II, version 1.1331 (T Gustafsson; Chalmers University of Technology).

\section{Biochemical measurements}

Venous blood was drawn from the participants after an overnight fast. Serum total cholesterol, HDL-cholesterol, LDLcholesterol and TAG concentrations were determined by the photometric enzymatic analysis. Insulin resistance was assessed with homeostatic model assessment of insulin resistance (HOMA-IR) and calculated using the following formula:

$$
\begin{aligned}
& \text { serum insulin }(\mathrm{mIU} / \mathrm{l}) \\
& \times \text { fasting plasma glucose }(\mathrm{mmol} / \mathrm{l}) / 22 \cdot 5^{(29)},
\end{aligned}
$$

where $1 \mathrm{mIU}$ insulin $=6 \mathrm{pmol}$ insulin.

\section{Framingham risk score and National Cholesterol Education Program's Adult Treatment Panel III}

The FRS gives an estimated risk of an individual for CHD over a period of 10 years. It is based on the Framingham Heart Study ${ }^{(21)}$. We calculated the risk score for each participant. Points were given for both sexes with separate risk score tables $^{(21)}$. The variables included were age, LDL-cholesterol, HDL-cholesterol, systolic or diastolic blood pressure (higher points were used), diabetes and smoking. According to the Framingham Heart Study, at the age of 30-34 years, the mean estimated risk of CHD (including angina pectoris) over 10 years is $3 \%$ for men and $<1 \%$ for women.

According to the NCEP-ATPIII, the presence of the metabolic syndrome is defined by the following five criteria, of 
which at least three should be present: waist circumference surrogating central fat $\geq 102 \mathrm{~cm}$ in men and $\geq 88 \mathrm{~cm}$ in women; blood pressure $>130 / 85 \mathrm{mmHg}$; fasting TAG concentration $\geq 1.7 \mathrm{mmol} / 1$ in men and $\geq 1.3 \mathrm{mmol} / 1$ in women; fasting glucose concentration $\geq 5.6 \mathrm{mmol} / \mathrm{l}$; HDL-concentration $<1.0 \mathrm{mmol} / 1$ in men and $<1.3 \mathrm{mmol} / 1$ in women ${ }^{(22,30)}$.

\section{Statistical methods}

The main outcome variables in the present study were the FRS (scale from -17 to 5 for women and from -8 to 5 for men) and the NCEP-ATPIII scoring (scale based on fulfilled criteria present, ranging from 0 to 5), and the secondary variables were systolic and diastolic blood pressure, total cholesterol, LDL-cholesterol, CCA-IMT and HOMA-IR. We used means and standard deviations to describe continuous variables, Student's $t$ test to compare the differences between groups and $\chi^{2}$ test for categorised variables. We used linear regression to test the effect of the duration of breast-feeding on each outcome variable. As possible confounders for the association between breast-feeding and risk factors of CVD, we tested sex, physical activity level (scale from 1 - no exercise, 2 - occasional physical activity, 3 - regular light physical activity such as gardening, 4 - regular light exercise such as walking to 5 - hard training more than twice per week), current dietary habits (from food records), current smoking (number of cigarettes smoked per d) and alcohol consumption (scale from 1 - no alcohol, 2 - alcohol less than once per month, 3 - up to twice per month, 4 - up to twice per week, 5 - three or four times per week to 6 - daily alcohol intake), level of education (scale from 1 - primary school to 6 - university degree), current anthropometric measurements and infancy-related factors (birth weight). We ended up using proportional intake of fat in the diet to distinguish between high- and low-fat diets accompanied with the intake of vitamin $\mathrm{C}$, a rough estimate of the amount of vegetables and fruits in the diet because data on micro-level were found to be more biased. Preliminary analyses were conducted to ensure no violation of the assumptions of normality, linearity and multicollinearity. Logarithmic transformations were used when needed. Linear regression was performed to evaluate the impact of the duration of breast-feeding on continuous variables (cardiovascular risk factors and FRS risk score). In model 1 , crude values were used. Model 2 was adjusted for sex, and model 3 was additionally adjusted for current lifestyle factors: physical activity; proportion of fats in the diet; vitamin C; smoking; alcohol consumption. Logistic regression was performed to assess the impact of the duration of breast-feeding on the probability to fulfil at least three criteria of the metabolic syndrome (NCEP-ATPIII). The same models were used as in linear regression analyses. SPSS Statistics 19.0 (IBM) was used for all analyses. A $P$ value $<0.05$ was considered as statistically significant.

\section{Results}

\section{Characteristics of the participants}

At 32 years of age, 158 participated in the present study (seventy-six males and eighty-two females), 66\% of the original cohort. No differences in the duration of breast-feeding between the participants (5.2 (SD 3.2) months) and nonparticipants (4.7 (SD 3.4) months) were detected (Student's $t$ test, $P=0.36)$, but, interestingly, birth weight $(P=0.035)$ and length $(P=0.003)$ were higher among non-participants. However, by 1 year of age, these differences attenuated between the participants and non-participants $(P=0 \cdot 11$ and $P=0 \cdot 14$, respectively; data not shown).

In the present study, three participants ( $2 \%)$, all women, were underweight (BMI $<18.5 \mathrm{~kg} / \mathrm{m}^{2}$ ), while $31 \%$ were overweight (BMI $25-30 \mathrm{~kg} / \mathrm{m}^{2}$ ) and $10 \%$ were obese (BMI $>30 \mathrm{~kg} / \mathrm{m}^{2}$ ). According to the NCEP-ATPIII criteria, $14 \%$ of men and $18 \%$ of women had central obesity. All anthropometric measures were higher in men (Table 1).

Table 1. Baseline demographic and clinical characteristics of the study cohort

(Mean values and standard deviations; percentages and $95 \%$ confidence intervals)

\begin{tabular}{|c|c|c|c|c|c|c|c|}
\hline & \multicolumn{2}{|c|}{ All $(n 158)$} & \multicolumn{2}{|c|}{ Males $(n 76)$} & \multicolumn{2}{|c|}{ Females ( $n$ 82) } & \multirow[b]{2}{*}{$P^{\star}$} \\
\hline & Mean & SD & Mean & SD & Mean & SD & \\
\hline \multicolumn{8}{|l|}{ Lifestyle factors } \\
\hline Weight (kg) & $75 \cdot 1$ & $14 \cdot 2$ & 83.7 & $12 \cdot 1$ & $67 \cdot 2$ & $11 \cdot 1$ & $<0.001$ \\
\hline Height $(\mathrm{cm})$ & $173 \cdot 6$ & $9 \cdot 0$ & $180 \cdot 3$ & $6 \cdot 5$ & $167 \cdot 5$ & $6 \cdot 1$ & $<0.001$ \\
\hline Waist circumference $(\mathrm{cm})$ & $84 \cdot 0$ & $11 \cdot 8$ & 89.9 & $10 \cdot 4$ & 78.5 & $10 \cdot 3$ & $<0.001$ \\
\hline $\mathrm{BMI}\left(\mathrm{kg} / \mathrm{m}^{2}\right)$ & $24 \cdot 8$ & $3 \cdot 8$ & $25 \cdot 7$ & $3 \cdot 3$ & $24 \cdot 0$ & 4.0 & 0.004 \\
\hline Smokers & & & & & & & 0.50 \\
\hline$\%$ & \multicolumn{2}{|c|}{33} & \multicolumn{2}{|c|}{36} & \multicolumn{2}{|c|}{30} & \\
\hline $95 \% \mathrm{Cl}$ & \multicolumn{2}{|c|}{$0.3,0.4 \dagger$} & \multicolumn{2}{|c|}{$0.2,0.5 \dagger$} & \multicolumn{2}{|c|}{$0.2,0.4 \dagger$} & \\
\hline Cigarettes/d (smokers) & $13 \cdot 0$ & 8.2 & $15 \cdot 5$ & $8 \cdot 8$ & $11 \cdot 1$ & $7 \cdot 1$ & 0.10 \\
\hline Alcohol consumption (scale 1-6) & 3.5 & 1.0 & $3 \cdot 8$ & $1 \cdot 1$ & $3 \cdot 3$ & 0.9 & 0.006 \\
\hline Proportion of fats in diet (\%) & 34.5 & $5 \cdot 8$ & $34 \cdot 1$ & $5 \cdot 7$ & 34.7 & $6 \cdot 0$ & 0.58 \\
\hline Vitamin C (mg) & $108 \cdot 8$ & 74.9 & 103.7 & 68.9 & $112 \cdot 9$ & 79.5 & 0.47 \\
\hline Current physical activity (scale 1-5) & $3 \cdot 2$ & 0.9 & $3 \cdot 1$ & 1.0 & $3 \cdot 3$ & 0.9 & 0.11 \\
\hline \multicolumn{8}{|l|}{ Infancy-related factors } \\
\hline Duration of breast-feeding (months) & $5 \cdot 2$ & $3 \cdot 2$ & 5.4 & 3.4 & 4.9 & $2 \cdot 9$ & 0.32 \\
\hline
\end{tabular}

*Based on Student's $t$ test.

† $95 \% \mathrm{Cl}$ for the difference. 
Table 2. Risk factors and vascular properties

(Mean values and standard deviations)

\begin{tabular}{|c|c|c|c|c|c|c|c|}
\hline & \multicolumn{2}{|c|}{ All } & \multicolumn{2}{|c|}{ Males } & \multicolumn{2}{|c|}{ Females } & \multirow[b]{2}{*}{$P^{*}$} \\
\hline & Mean & SD & Mean & SD & Mean & SD & \\
\hline \multicolumn{8}{|l|}{ Biochemical markers } \\
\hline Cholesterol $(\mathrm{mmol} / \mathrm{l})$ & 4.5 & 0.9 & 4.8 & 1.0 & 4.3 & 0.7 & $<0.001$ \\
\hline HDL-cholesterol (mmol/l) & $2 \cdot 6$ & 1.6 & 1.4 & 0.3 & 1.7 & 0.4 & $<0.001$ \\
\hline LDL-cholesterol (mmol/l) & $2 \cdot 4$ & 0.8 & $2 \cdot 8$ & 0.8 & $2 \cdot 1$ & 0.6 & $<0.001$ \\
\hline $\mathrm{TAG}(\mathrm{mmol} / \mathrm{l})$ & 1.0 & 1.0 & 1.3 & 1.4 & 0.8 & 0.4 & 0.006 \\
\hline HOMA-IR (mmol/l) & $1 \cdot 3$ & 0.8 & 1.5 & 0.9 & $1 \cdot 2$ & 0.6 & 0.013 \\
\hline Fasting glucose $(\mathrm{mmol} / \mathrm{l})$ & $5 \cdot 2$ & 0.6 & 5.4 & 0.6 & $5 \cdot 0$ & 0.5 & $<0.001$ \\
\hline Fasting insulin $(\mathrm{mmol} / \mathrm{l})$ & $5 \cdot 6$ & $3 \cdot 1$ & $6 \cdot 0$ & 3.6 & $5 \cdot 2$ & $2 \cdot 5$ & 0.14 \\
\hline \multicolumn{8}{|l|}{ Blood pressure } \\
\hline Systolic blood pressure $(\mathrm{mmHg})$ & $120 \cdot 0$ & 13.6 & 126.5 & $12 \cdot 8$ & $113 \cdot 8$ & 11.4 & $<0.001$ \\
\hline Diastolic blood pressure $(\mathrm{mmHg})$ & 73.5 & 8.5 & $76 \cdot 1$ & $8 \cdot 8$ & $71 \cdot 1$ & 7.5 & $<0.001$ \\
\hline \multicolumn{8}{|l|}{ Vascular properties (CCA) } \\
\hline Intima-media thickness (mm) & 0.46 & 0.08 & 0.48 & 0.08 & 0.44 & 0.06 & 0.051 \\
\hline \multicolumn{8}{|l|}{ Risk scores } \\
\hline Framingham risk score (range -17 to 5 ) & -8.0 & $6 \cdot 4$ & -1.5 & $2 \cdot 6$ & $-14 \cdot 1$ & $2 \cdot 7$ & $<0.001$ \\
\hline NCEP-ATPIII (range 0-5) & 0.8 & $1 \cdot 1$ & 1.1 & $1 \cdot 2$ & 0.4 & 0.8 & $<0.001$ \\
\hline
\end{tabular}

Alcohol consumption more than twice per week was reported by $13 \%$ of the participants. Compared with women, men had significantly higher alcohol consumption (Student's $t$ test, $P=0 \cdot 006$; Table 1). The frequency of the regular usage of tobacco products was $33 \%$. Of the participants, $73 \%$ reported current physical activity at least twice per week. Participants were highly educated as $78 \%$ had graduated from high school and $57 \%$ had a university degree. Of the women participants, $52 \%$ had at least one child. According to the $3 \mathrm{~d}$ food records, men received on average $34 \%$ of their daily energy from fats and 18\% from proteins, while the corresponding estimates in women were 35 and $17 \%$, respectively. The mean intakes of vitamin $\mathrm{C}$ were $104 \mathrm{mg}$ in men and $113 \mathrm{mg}$ in women.

\section{Characteristics of the cardiovascular outcomes and risk scores}

None of the participants used lipid-lowering medication, although $26 \%$ had total cholesterol concentration above $5.0 \mathrm{mmol} / 1,23 \%$ had LDL-concentration above $3.0 \mathrm{mmol} / \mathrm{l}$, $7 \%$ had TAG concentration above $1.7 \mathrm{mmol} / \mathrm{l}$, and $9 \%$ of men had HDL-concentration below $1.0 \mathrm{mmol} / \mathrm{l}$ and $6 \%$ of women below $1.3 \mathrm{mmol} / 1$ (NCEP-ATPIII cut-off points). The measure of insulin resistance HOMA-IR was higher in men than in women (Table 2).

At 32 years of age, the mean systolic blood pressure was $120 \mathrm{mmHg}$ and the mean diastolic blood pressure was $73.5 \mathrm{mmHg}$. According to the NCEP-ATPIII cut-off points, $25 \%$ had systolic blood pressure above $130 \mathrm{mmHg}$ and $10 \%$ had diastolic blood pressure above $85 \mathrm{mmHg}$. Furthermore, two participants with elevated diastolic blood pressure had systolic pressure below $130 \mathrm{mmHg}$. Only one participant reported the use of antihypertensive medication; however, despite the treatment, he was hypertensive. The CCA-IMT tended to be higher in men (Table 2).

According to the FRS, twenty-two (29\%) men had at least $3 \%$ risk of CHD over 10 years. For the remaining men, the risk was lower. None of the women had a notably elevated risk compared with the average risk at this age. The criteria for the metabolic syndrome according to the NCEP-ATPIII were met by two women and twelve men (Fig. 1). Nine of these also had an elevated risk of CHD within 10 years.

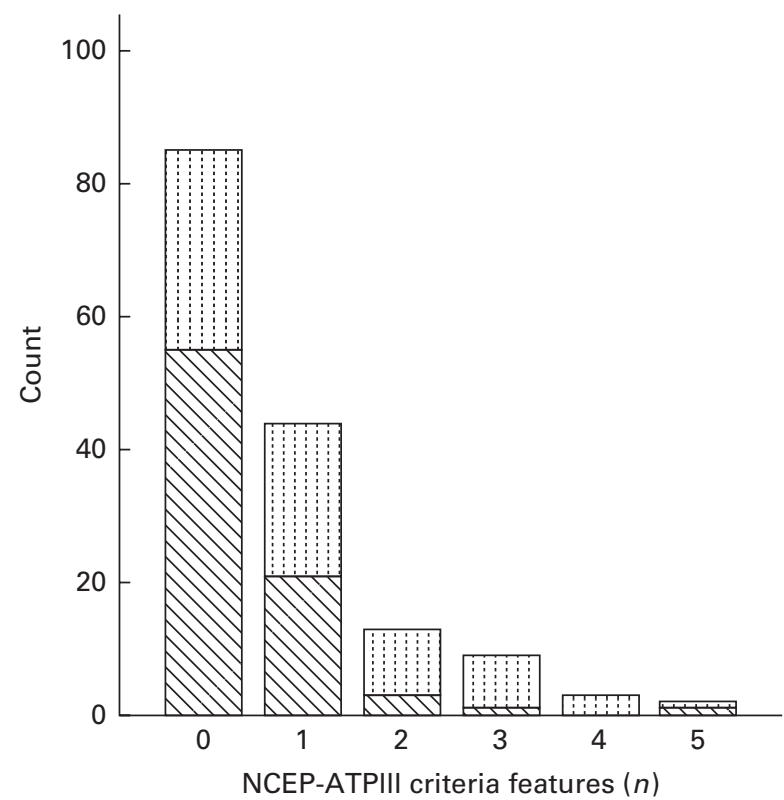

Fig. 1. Clustering of metabolic criteria features in the participants defined according to the National Cholesterol Education Program's Adult Treatment Panel III (NCEP-ATPIII). The metabolic syndrome requires the presence of three or more features. The observed features were central obesity, glucose intolerance or diabetes, hypertension and dyslipidaemia (HDL and TAG). Sex: $\square$, male; $\mathbb{N}$, female. 


\section{Breast-feeding and cardiovascular risk factors}

Pearson's correlation showed that the duration of breastfeeding did not have significant associations with FRS, NCEP-ATPIII scoring, vascular variables, blood pressure or lipid profile as the correlations were within the range of $0 \cdot 01-0 \cdot 06$. Table 3 presents the associations between the duration of breast-feeding and all the outcomes as analysed with linear regression. Model 1 represents crude values. The results are shown in relation to the absolute duration of breastfeeding. Again, the duration of breast-feeding did not have significant associations with FRS, blood pressure, CCA-IMT or cholesterol levels ( $P>0.5$ for all). In model 2 , the values were adjusted for sex, which seems to be the most important predictor of CVD risk. In model 3, values were additionally adjusted for current physical activity, dietary proportion of fat and vitamin $\mathrm{C}$, number of cigarettes per $\mathrm{d}$ and alcohol consumption. These adjustments did not influence the results. After all the adjustments, model 3 explained $85 \%$ of the variance in the FRS.

Due to a skewed variation of the NCEP-ATPIII in the cohort (Fig. 1), the association with the duration of breast-feeding was tested using logistic regression. Subjects who fulfilled at least three of the NCEP-ATPIII criteria were recoded as 1 ( $n$ 14, increased risk, multiple risk factors) and others with 0. In model 1 , the OR characterising the impact of a 1-month increase in the duration of breast-feeding was 0.96 (95\% CI $0.8,1.1)$, in model 2 adjusting for sex, OR was $0.95(95 \%$ CI $0 \cdot 8,1 \cdot 1)$ and in model 3 containing all confounders, OR was $0.95(95 \%$ CI $0 \cdot 8,1 \cdot 1)$

\section{Discussion}

We demonstrate here that cardiovascular risk in adulthood is not influenced by the duration of breast-feeding in subjects born at term and appropriate for gestational age. The present findings were not modified by the current lifestyle factors.
Male sex was the most important independent predictor of CVD. Clustered FRS, an estimate of 10-year CHD risk, was not related to the duration of breast-feeding.

The present study population is a unique birth cohort that has been followed up particularly closely during the first 12 months by the same physician, and the information on milk feeding has been obtained during frequent visits in infancy, thus avoiding recall bias. The prospective follow-up period of 32 years can be considered to be exquisitely long. The additional strength of the present study is the carefully collected data on confounding factors, such as current lifestyle factors, diet and physical activity, which were obtained in a structured fashion with a questionnaire and completed by interview by one of the authors. Originally, the collected cohort was homogeneous, as all babies were born at the same delivery hospital at term, their birth weight was above $3000 \mathrm{~g}$ and complementary foods were introduced to all at 3.5 months with the same guidelines. Also, the environments, including public primary and secondary schools, were similar for all participants, as they were native Finns with a similar cultural background. In our cohort, no cardiovascular events were diagnosed, as myocardial infarction or angina pectoris is still very rare by 32 years of age. Therefore, our approach was to investigate the association of infant feeding with the risk of cardiovascular events within 10 years. For our purposes, the FRS and NCEP-ATPIII metabolic syndrome criteria points (which exploit multiple CVD risk factors) were suitable and novel compared with prior studies.

Our cohort consisted mostly of relatively well-educated participants, which may have influenced the results. Breastfeeding is known to associate with higher socio-economic status and healthier lifestyle overall, and, accordingly, the results may not be representative for all social classes. In addition, the cohort size was limited. Therefore, some smaller potential effects of breast-feeding might not become notable

Table 3. Various cardiovascular risk variables*

(Regression coefficients and 95\% confidence intervals)

\begin{tabular}{|c|c|c|c|c|c|c|c|}
\hline $\begin{array}{l}\text { Exposure: duration } \\
\text { of breast-feeding } \\
\text { (months) }\end{array}$ & $\begin{array}{c}\text { FRS } \\
\text { (range }-17 \text { to } 5 \text { ) }\end{array}$ & $\begin{array}{c}\text { Systolic blood } \\
\text { pressure }(\mathrm{mmHg})\end{array}$ & $\begin{array}{c}\text { Diastolic blood } \\
\text { pressure }(\mathrm{mmHg})\end{array}$ & $\begin{array}{l}\text { Cholesterol } \\
(\mathrm{mmol} / \mathrm{l})\end{array}$ & $\begin{array}{l}\text { LDL-cholesterol } \\
(\mathrm{mmol} / \mathrm{l})\end{array}$ & $\begin{array}{l}\text { CCA-IMT } \\
(\mathrm{mm})\end{array}$ & $\begin{array}{l}\text { HOMA-IR } \\
(\mathrm{mmol} / \mathrm{l}) \dagger \\
\end{array}$ \\
\hline \multicolumn{8}{|l|}{ Model $1 \ddagger$} \\
\hline$\beta$ & 0.06 & -0.02 & -0.05 & -0.01 & 0.01 & 0.04 & -0.02 \\
\hline $95 \% \mathrm{Cl}$ & $-0.24,0.48$ & $-0.78,0.67$ & $-0.57,0.33$ & $-0.05,0.04$ & $-0.04,0.04$ & $-0.01,0.01$ & $-0.03,0.03$ \\
\hline$P$ & 0.50 & 0.85 & 0.60 & 0.92 & 0.95 & 0.75 & 0.81 \\
\hline \multicolumn{8}{|l|}{ Model 2§ } \\
\hline$\beta$ & -0.02 & -0.05 & -0.07 & -0.03 & -0.03 & 0.02 & -0.03 \\
\hline $95 \% \mathrm{Cl}$ & $-0.18,0.11$ & $-0.90,0.37$ & $-0.61,0.24$ & $-0.05,0.04$ & $-0.05,0.03$ & $-0.01,0.01$ & $-0.04,0.02$ \\
\hline$P$ & 0.62 & 0.47 & 0.40 & 0.68 & 0.70 & 0.87 & 0.68 \\
\hline \multicolumn{8}{|l|}{ Model 3\| } \\
\hline$\beta$ & -0.02 & -0.06 & -0.07 & -0.02 & -0.01 & 0.02 & -0.05 \\
\hline $95 \% \mathrm{Cl}$ & $-0.19,0.09$ & $-0.90,0.40$ & $-0.63,0.23$ & $-0.05,0.04$ & $-0.04,0.04$ & $-0.01,0.01$ & $-0.04,0.02$ \\
\hline$P$ & 0.47 & 0.42 & 0.36 & 0.81 & 0.88 & 0.89 & 0.58 \\
\hline
\end{tabular}

FRS, Framingham risk score; CCA-IMT, common carotid artery intima-media thickness; HOMA-IR, homeostatic model assessment.

* The value of the coefficient characterises the impact of 1-month increase in the duration of breast-feeding on cardiovascular risk variables.

$\dagger$ For HOMA-IR, logarithmic transformation (natural logarithm) was utilised.

$¥$ Model 1 shows crude values.

$\S$ Model 2 is adjusted for sex.

\| Model 3 is same as model 2 plus adjusted for current physical activity, proportion of fats in the diet, dietary vitamin $\mathrm{C}$, alcohol consumption and number of cigarettes per $\mathrm{d}$ (smoking is used as a covariate in all the outcome variables except the FRS which already includes smoking as one of its components). 
in our cohort. However, even if the end points of the CI may be over 10-fold when compared with the coefficient of breastfeeding duration $\beta$ itself (Table 3 ), they are still sufficiently narrow to enable considering the effect of the duration of breast-feeding as minor. Considering the FRS for model 3, the lower endpoint of the CI is $-0 \cdot 19$; hence, over 5 -month increase in the duration of breast-feeding would be required to decrease the sum of the FRS by one point, which corresponds to about $1 \%$ decrease in the 10 -year CHD risk.

The role of breast-feeding as a potential determinant of individual cardiovascular risk factors has been of interest in several studies. A meta-analysis by Owen et ll $^{(31)}$ included thirty-seven studies on the effect of infant feeding mode on total cholesterol and LDL-cholesterol. Breast-fed infants were compared with bottle-fed infants, and no differences were detected in their total cholesterol or LDL-cholesterol levels at adolescence, but at adulthood ( $>17$ years of age), those who received breast milk had a lower mean cholesterol level of $0 \cdot 18 \mathrm{mmol} / \mathrm{l}^{(31)}$. Other studies with an extended follow-up up to middle age have reported no associations between breast-feeding and later blood pressure or lipid profile at 41 years of age ${ }^{(15,32)}$. These reports support the present findings with no clinically relevant association discovered between infant feeding and the risk of CVD at 32 years of age.

In developed countries, higher socio-economic class, mature maternal age, lower maternal BMI and, at the same time, the choice of healthier lifestyle in general are known to associate with the mother's intention to breast-feed and also to the actual duration of breast-feeding ${ }^{(33-35)}$, and, in turn, the same factors contribute to the offspring's health. Accordingly, numerous confounders confuse the interpretation of the effect of breast-feeding on adult cardiovascular health. Therefore, to evaluate the true effect of infant feeding, we collected data on lifestyle factors and dietary habits, and used these confounders as covariates in statistical analysis. The Promotion of Breast-feeding Intervention Trial (PROBIT) has been designed to anticipate the control of confounders. This intervention study was scheduled by bringing the WHO Baby Friendly Hospital Initiative training to promote breastfeeding at randomised birth hospitals in Belarus. PROBIT has increased the duration of exclusive breast-feeding in these hospitals. Only breast-fed infants were included to PROBIT, and in their recent evaluation (2013) at 11 years of age, no difference has been reported in body composition and obesity between those exclusively breast-fed for 3 months and those with longer breast-feeding ${ }^{(36)}$.

In reported studies, the association of breast-feeding with cardiovascular risk factors has been controversial, as variables showing beneficial effects vary between studies: they might have reported beneficial effects on one and no effect on another risk factor and vice versa with no consistency ${ }^{(8,32,37)}$. Large cohorts enable the detection of small significant changes between study groups. Typically, this raises a question about the clinical importance or even the meaning to public health. Based on two large simultaneous meta-analyses involving seventeen and twenty-four studies and altogether $>17000$ participants, breast-feeding reduced systolic blood pressure by $1.1-1.4 \mathrm{mmHg}$ later in life when compared with those formula fed in infancy. The reduction in diastolic blood pressure was moderate $(0.5 \mathrm{mmHg})$. The authors have suggested that studies of small cohorts $(n<300)$ reported larger mean differences between infant-feeding groups, and these studies are subject to a chance finding ${ }^{(12,13)}$. Similarly, in the Mater-University Study of Pregnancy and its outcomes in Australia with nearly 4000 participants, the authors have reported a $0.69 \mathrm{mmHg}$ lower systolic blood pressure at age 5 years among participants breast-fed over 6 months, compared with those who were breast-fed for a shorter duration ${ }^{(9)}$. The European Youth Heart Study of over 2000 participants aged 9-15 years showed a $1.7 \mathrm{mmHg}$ reduction in systolic blood pressure in favour of breast-feeding over formula feeding ${ }^{(8)}$.

The effect of breast-feeding on serum cholesterol and TAG has been studied in the Framingham Third Generation Study: being breast-fed as an infant was associated with higher HDL-cholesterol levels at the age of 41 years, but no difference in other lipid levels or blood pressure was recognised, as mentioned earlier ${ }^{(32)}$. Khan et al. ${ }^{(38)}$ reported improved microvascular function in 10-14-year-olds who had been breast-fed. In a Finnish study, men aged 24-39 years who had been breast-fed had better brachial endothelial function compared with those initially formula fed; in women, no differences were observed ${ }^{(10)}$. Finally, only a few studies have reported the non-beneficial effects of breast-feeding, which may reflect publication bias. For example, Leeson et $a l .{ }^{(17)}$ reported reduced arterial distensibility with longer breast-feeding. Non-invasive ultrasonic measurements of arterial distensibility, flow-mediated endothelial function and the thickening of arterial IMT are potential hallmarks of subclinical $\mathrm{CVD}^{(39)}$. These measurements are mostly used by researchers and are not yet in clinical practice.

\section{Conclusion}

In the present long-term prospective study from infancy to adulthood, we found a very weak association between the duration of breast-feeding and cardiovascular risk factors in adulthood. The present findings were not modified by the current lifestyle factors, including physical activity, dietary habits, smoking and alcohol consumption. Male sex seems to be the most predictive determinant of the increased risk of CVD. The associations in previous studies have been weak as well, and the clinical significance of the effects is questionable at both the individual and public health levels. As breast-feeding has many other beneficial aspects to infants, we, however, do recommend mothers to breast-feed their babies.

\section{Supplementary material}

To view supplementary material for this article, please visit http://dx.doi.org/10.1017/S0007114513003346

\section{Acknowledgements}

The authors acknowledge adjunct Professor Hannu Rita from the Department of Forest Resource Management (Statistics and Methodology), University of Helsinki for statistical 
expertise. The authors also thank Minna Savolainen, RN, for the help in the data collection and Ulla Vesander, RD, for analysing the food records, both from the Children's Hospital, Helsinki University Central Hospital.

The present study was supported by grants from the following organisations in Helsinki, Finland: Foundation for Pediatric Research; Academy of Finland; Folkhälsan Research Foundation; Helsinki University Central Hospital Research Funds; Sigrid Juselius Foundation; Ida Montin Foundation; Yrjö Jahnsson Foundation. The founders had no role in the study design, data collection and analysis, decision to publish or preparation of the manuscript.

The authors' contributions are as follows: S. P., M. Ta., O. M., M. K., U. M. S.-P. and M. Tu. took part in the planning of the study design; S. P. was responsible for the data collection at the 32-year follow-up; S. P., M. Ta., H. V. and M. Tu. were responsible for the analysis and interpretation of the data; S. P. wrote the manuscript with the assistance of M. Ta., H. V. and M. Tu. All authors took part on the manuscript preparation and contributed intellectually to the writing. Each author read and approved the contents of the manuscript.

None of the authors has any conflict of interest.

\section{References}

1. Barker DJ (1995) Fetal origins of coronary heart disease. BMJ 311, 171-174.

2. Barker DJ, Gluckman PD, Godfrey KM, et al. (1993) Fetal nutrition and cardiovascular disease in adult life. Lancet 341, 938-941.

3. Banci M, Saccucci P, Dofcaci A, et al. (2009) Birth weight and coronary artery disease. The effect of gender and diabetes. Int J Biol Sci 5, 244-248.

4. Eriksson JG (2011) Early growth and coronary heart disease and type 2 diabetes: findings from the Helsinki Birth Cohort Study (HBCS). Am J Clin Nutr 94, 6 Suppl., 1799S-1802S

5. Owen CG, Whincup PH, Kaye SJ, et al. (2008) Does initial breastfeeding lead to lower blood cholesterol in adult life? A quantitative review of the evidence. Am J Clin Nutr 88, 305-314.

6. Kaikkonen JE, Mikkilä V, Magnussen CG, et al. (2013) Does childhood nutrition influence adult cardiovascular disease risk? - Insights from the Young Finns Study. Ann Med 45, $120-128$.

7. Martin RM, Ness AR, Gunnell D, et al. (2004) Does breastfeeding in infancy lower blood pressure in childhood? The Avon Longitudinal Study of Parents and Children (ALSPAC). Circulation 109, 1259-1266.

8. Lawlor DA, Riddoch CJ, Page AS, et al. (2005) Infant feeding and components of the metabolic syndrome: findings from the European Youth Heart Study. Arch Dis Child 90, $582-588$

9. Lawlor DA, Najman JM, Sterne J, et al. (2004) Associations of parental, birth, and early life characteristics with systolic blood pressure at 5 years of age: findings from the MaterUniversity study of pregnancy and its outcomes. Circulation 110, 2417-2423.

10. Järvisalo MJ, Hutri-Kähönen N, Juonala M, et al. (2009) Breast feeding in infancy and arterial endothelial function later in life. The Cardiovascular Risk in Young Finns Study. Eur J Clin Nutr 63, 640-645.
11. Martin RM, Ebrahim S, Griffin M, et al. (2005) Breastfeeding and atherosclerosis: intima-media thickness and plaques at 65-year follow-up of the Boyd Orr cohort. Arterioscler Thromb Vasc Biol 25, 1482-1488.

12. Owen CG, Whincup PH, Gilg JA, et al. (2003) Effect of breast feeding in infancy on blood pressure in later life: systematic review and meta-analysis. BMJ 327, 1189-1195.

13. Martin RM, Gunnell D \& Smith GD (2005) Breastfeeding in infancy and blood pressure in later life: systematic review and meta-analysis. Am J Epidemiol 161, 15-26.

14. Verier CM, Duhamel A, Beghin L, et al. (2011) Breastfeeding in infancy is not associated with inflammatory status in healthy adolescents. J Nutr 141, 411-417.

15. Rudnicka AR, Owen CG \& Strachan DP (2007) The effect of breastfeeding on cardiorespiratory risk factors in adult life. Pediatrics 119, e1107-e1115.

16. de Jonge LL, van Osch-Gevers L, Geelhoed JJ, et al. (2010) Breastfeeding is not associated with left cardiac structures and blood pressure during the first two years of life. The Generation R Study. Early Hum Dev 86, 463-468.

17. Leeson CP, Kattenhorn M, Deanfield JE, et al. (2001) Duration of breast feeding and arterial distensibility in early adult life: population based study. BMJ 322, 643-647.

18. Pirilä S, Saarinen-Pihkala UM, Viljakainen H, et al. (2012) Breastfeeding and determinants of adult body composition: a prospective study from birth to young adulthood. Horm Res Paediatr 77, 281-290.

19. Saarinen UM \& Siimes MA (1979) Role of prolonged breast feeding in infant growth. Acta Paediatr Scand 68, 245-250.

20. Touboul PJ, Labreuche J, Vicaut E, et al. (2005) Carotid intimamedia thickness, plaques, and Framingham risk score as independent determinants of stroke risk. Stroke 36, 1741-1745.

21. Wilson PW, D'Agostino RB, Levy D, et al. (1998) Prediction of coronary heart disease using risk factor categories. Circulation 97, 1837-1847.

22. Olufadi R \& Byrne CD (2008) Clinical and laboratory diagnosis of the metabolic syndrome. J Clin Pathol 61, 697-706.

23. Saarinen UM (1978) Need for iron supplementation in infants on prolonged breast feeding. J Pediatr 93, 177-180.

24. Saarinen UM \& Siimes MA (1978) Developmental changes in red blood cell counts and indices of infants after exclusion of iron deficiency by laboratory criteria and continuous iron supplementation. J Pediatr 92, 412-416.

25. Saarinen UM \& Siimes MA (1978) Serum ferritin in assessment of iron nutrition in healthy infants. Acta Paediatr Scand 67, 745-751.

26. Saarinen UM \& Siimes MA (1979) Iron absorption from breast milk, cow's milk, and iron-supplemented formula: an opportunistic use of changes in total body iron determined by hemoglobin, ferritin, and body weight in 132 infants. Pediatr Res 13, 143-147.

27. Hultin H, Opas R \& Sarna S (1974) Breast feeding of babies in Finland in the seventies. Katilolehti 79, 365-372.

28. Pirilä S, Taskinen M, Viljakainen H, et al. (2011) Infant milk feeding influences adult bone health: a prospective study from birth to 32 years. PLOS ONE 6, e19068.

29. Haffner SM, Kennedy E, Gonzalez C, et al. (1996) A prospective analysis of the HOMA model. The Mexico City Diabetes Study. Diabetes Care 19, 1138-1141.

30. Grundy SM, Cleeman JI, Daniels SR, et al. (2005) Diagnosis and management of the metabolic syndrome: an American Heart Association/National Heart, Lung, and Blood Institute Scientific Statement. Circulation 112, 2735-2752.

31. Owen CG, Whincup PH, Odoki K, et al. (2002) Infant feeding and blood cholesterol: a study in adolescents and a systematic review. Pediatrics 110, 597-608. 
32. Parikh NI, Hwang SJ, Ingelsson E, et al. (2009) Breastfeeding in infancy and adult cardiovascular disease risk factors. $A m J$ Med 122, 656e1-663e1.

33. Ludvigsson JF \& Ludvigsson J (2005) Socio-economic determinants, maternal smoking and coffee consumption, and exclusive breastfeeding in 10205 children. Acta Paediatr 94, 1310-1319.

34. Grjibovski AM, Ehrenblad B \& Yngve A (2008) Infant feeding in Sweden: socio-demographic determinants and associations with adiposity in childhood and adolescence. Int Breastfeed J 3, 23.

35. Oddy WH, Li J, Landsborough L, et al. (2006) The association of maternal overweight and obesity with breastfeeding duration. J Pediatr 149, 185-191.
36. Martin RM, Patel R, Kramer MS, et al. (2013) Effects of promoting longer-term and exclusive breastfeeding on adiposity and insulin-like growth factor-I at age 11.5 years: a randomized trial. JAMA 309, 1005-1013.

37. Ravelli AC, van der Meulen JH, Osmond C, et al. (2000) Infant feeding and adult glucose tolerance, lipid profile, blood pressure, and obesity. Arch Dis Child 82, 248-252.

38. Khan F, Green FC, Forsyth JS, et al. (2009) The beneficial effects of breastfeeding on microvascular function in 11- to 14-year-old children. Vasc Med 14, 137-142.

39. Lorenz MW, Markus HS, Bots ML, et al. (2007) Prediction of clinical cardiovascular events with carotid intima-media thickness: a systematic review and meta-analysis. Circulation 115, 459-467. 